\title{
Resonant acousto-optics of microcavity polaritons
}

\author{
A.L. Ivanov \\ Cardiff University, Department of Physics and Astronomy, Queen's Buildings, 5 The Parade, Cardiff CF24 3 YB, Wales UK \\ P.B. Littlewood \\ University of Cambridge, Department of Physics, TCM Group, Cavendish Laboratory, Cambridge CB3 0HE, UK
}

(December 6, 2002)

\begin{abstract}
We propose and analyze theoretically a resonant acousto-optic Stark effect for microcavity (MC) polaritons parametrically driven by a surface acoustic wave. For GaAs-based microcavities our scheme "acoustic pumping - optical probing" deals with surface acoustic waves of frequency $\nu_{\text {SAW }} \simeq$ $0.5-3 \mathrm{GHz}$ and intensity $I_{\mathrm{SAW}} \simeq 0.1-10 \mathrm{~mW} / \mathrm{mm}$. The acoustically-induced stop gaps in the MC polariton spectrum drastically change the optical response of MC polaritons. Because an acoustically pumped intrinsic semiconductor microcavity remains in its ground electronic state, no many-body effects screen and weaken the resonant acousto-optic Stark effect. In the meantime, this allows us to work out an exactly-solvable model for resonant acousto-optics of MC polaritons which deals with giant acousto-optical nonlinearities. Finally, we discuss possible applications of the proposed resonant acoustic Stark effect for optical modulation and switching and describe an acousto-optic device based on a (GaAs) microcavity driven by a surface acoustic wave.
\end{abstract}

71.35.-y, 42.65.-k, 63.20.Ls

\section{INTRODUCTION}

The science of acousto-optics is a well-established field that relies on the modulation of the dielectric constant of a material by a pattern of sound waves to produce diffraction of incident light [1-3]. In the conventional acousto-optics, the principle effect on the dielectric constant arises just from the modulation of the density; correspondingly the modulation is weak and deals with small nonresonant acousto-optical nonlinearities. Most of the light on the sample will pass through undiffracted.

The resonant acousto-optics $[4,5]$ we develop here for microcavity polaritons associated with quantum well (QW) excitons deals with giant acousto-optical nonlinearities mediated by the QW excitonic resonance. In this case the momentum-energy matching condition, necessary for appearance of the acoustically-induced optical stop gaps, occurs for optical frequencies nearly resonant with QW excitons. As a result, the acousto-optical susceptibility is enhanced by several orders of magnitude in comparison to conventional applications, both due to small detuning from the excitonic resonance and the relatively small damping constants relevant to QW excitons and MC photons (for GaAs-based MCs a typical polaritonic linewidth is less than $0.5 \mathrm{meV}$ ).

Linear and nonlinear optics of MC polaritons are now a well-established field (see for example [6-14]). There are several points in favour of the resonant acoustooptics of MC polaritons: (i) a well-controlled in-plane wave interaction in microcavities, (ii) a compatibility of semiconductor microcavities with surface acoustic wave (SAW) technique, and (iii) a possibility to realize onedimensional geometry for resonant, SAW-mediated interaction of two counter-propagating MC polaritons.
In this article, we discuss how SAW and microcavity technologies can be combined to make devices where the SAW can produce a tunable and narrow optical band gap, while being driven at modest powers. An attractive feature of this technique is that the process does not involve real excitation of excitons or electron-hole pairs. The strong resonant coupling produces an interaction length of typically $100 \mu \mathrm{m}$ or less, making the process suitable for device applications. Our analysis is provided here for appropriate parameters for GaAs at low temperatures, since this is the material for which both the technologies of SAW and microcavity are well developed. However, the phenomenon occurs in any material with a sharp resonance in the optical spectrum and a strong piezo-electric or deformation potential coupling; which includes organic semiconductors, other semiconductors such as CdTe or $\mathrm{ZnSe}$, and rare-earth-doped glass. In many of these materials, the effects are much larger than in GaAs, and will be substantial even at room temperature.

The discovery of the optical Stark effect mediated by an excitonic resonance in semiconductors and semiconductor nanostructures [15-17] initiated numerous attempts to apply the effect for ultra-fast light-light processing. Later on, however, it was realized that the excitonic optical Stark effect requires high-intensity laser fields, $I_{\text {opt }} \simeq 0.1-10 \mathrm{GW} / \mathrm{cm}^{2}$, so that for a pump electromagnetic pulse nearly resonant with excitons it is practically impossible to avoid generation of real, incoherent electrons and holes, keeping a semiconductor under purely virtual optical excitation. As a result, the incoherent scattering rate of excitons strongly increases with increasing $I_{\mathrm{opt}}$ and considerably relaxes the optically-induced spectral changes. Furthermore, a highintensity laser pulse, which gives rise to the optical Stark 
effect, weakens the exciton-photon interaction and relaxes the excitonic states, due to the Coulomb interaction between photogenerated virtual (and real) excitons and due to Pauli blocking of the relevant single-electron states. The above arguments explain why the excitonmediated optical Stark effect, which refers to the scheme "optical pumping - optical probing", cannot practically be used for optoelectronic applications.

By the same arguments, particular realizations of the exciton-mediated optical Stark effect, due to excitonic molecules [18-21] or due to exciton-phonon interaction [22-24], remain mainly a question of interesting fundamental science with little practical application as yet. For example, the acoustic phonoriton spectrum, which gives rise to the phonon-mediated Stark effect, has recently been measured and analyzed for orthoexcitons in $\mathrm{Cu}_{2} \mathrm{O}$ driven by an optical field of intensity $I_{\mathrm{opt}} \simeq$ $0.1-1 \mathrm{GW} / \mathrm{cm}^{2}[25,26]$. In spite of relatively strong coupling of excitons with acoustic phonons in GaAs, one cannot realize the phonon-mediated optical Stark effect in this semiconductor: the large Bohr radius of excitons does not allow the use of high $I_{\text {opt }}$ without the modification and eventual removal of the excitonic states.

The resonant acousto-optics, which in this paper we develop for MC polaritons, deals with an alternative scheme of the dynamic Stark effect: acoustically driven MC polaritons, associated with QW excitons, probed by weak light (acoustically-induced Stark effect). Because an acoustically pumped intrinsic semiconductor microcavity remains in its ground electronic state, no manybody effects screen the resonant acousto-optic Stark effect and weaken the QW exciton - MC photon interaction. Being applied to GaAs-based MCs, the proposed scheme "acoustic pumping - optical probing" effectively exploits both the relatively strong exciton-phonon piezoelectric interaction and the considerable exciton-photon coupling relevant to bulk GaAs [27]. As we show below, the above processing scheme requires moderate-intensity acoustic and low-intensity electromagnetic waves but can still operate in a picosecond time domain (the latter needs a particular design of a GaAs microcavity driven by a surface acoustic wave, see section 4 ). Thus the resonant acousto-optic Stark effect for MC polaritons takes all the advantages of the excitonic optical Stark effect, but needs much smaller operating intensities.

In the last decade SAWs were widely applied mainly to study electron and hole transport in GaAs-based QW structures. Surface acoustic waves with frequency $\nu_{\mathrm{SAW}}=2-9 \mathrm{GHz}$ have been successfully used to investigate the fractional quantum Hall effect for a quasi-two-dimensional electron system in lowtemperature GaAs/AlGaAs heterostructures [28,29]. A single-electron acoustoelectric current induced by SAWs with $\nu_{\mathrm{SAW}}=2-3 \mathrm{GHz}$ in a quasi-one-dimensional (quasi-1D) GaAs channel has been observed and analyzed [30,31]. Acoustically-induced ionization of photogenerated QW excitons and subsequent long-distance acoustoelectric transport of free electrons and holes have been detected in InGaAs/GaAs QWs driven by a SAW with $\nu_{\mathrm{SAW}}=0.1-0.9 \mathrm{GHz}[32-34]$. The surface acoustical waves with experimentally accessible parameters, frequency $\nu_{\mathrm{SAW}} \lesssim 2-3 \mathrm{GHz}$ and moderate intensity $I_{\text {SAW }} \lesssim 10 \mathrm{~mW} / \mathrm{mm}$, are also very suitable for effective realization of the resonant acousto-optics of MC polaritons. Note that in this case the SAW intensities are much less than those used to ionized the QW excitons $[32,33]$.

\section{MICROCAVITY POLARITONS PARAMETRICALLY DRIVEN BY THE COHERENT ACOUSTIC FIELD}

Our model deals with a QW microcavity parametrically driven by an in-plane propagating coherent acoustic wave of frequency $\Omega_{\mathbf{k}}^{\mathrm{ac}}$, wavevector $\mathbf{k}$ and intensity $I_{\mathrm{ac}}$. In this case the Hamiltonian of QW excitons and in-plane $\mathrm{MC}$ photons is given by

$$
\begin{aligned}
H & =\sum_{\mathbf{p}_{\|}}\left[\hbar \omega_{\mathbf{p}_{\|}}^{\mathrm{X}} B_{\mathbf{p}_{\|}}^{\dagger} B_{\mathbf{p}_{\|}}+\hbar \omega_{\mathbf{p}_{\|}}^{\mathrm{MC}} \alpha_{\mathbf{p}_{\|}}^{\dagger} \alpha_{\mathbf{p}_{\|}}\right. \\
& +i \hbar \frac{\Omega_{\mathbf{X}}^{\mathrm{MC}}}{2}\left(\alpha_{\mathbf{p}_{\|}}^{\dagger} B_{\mathbf{p}_{\|}}-B_{\mathbf{p}_{\|}}^{\dagger} \alpha_{\mathbf{p}_{\|}}\right) \\
& \left.+i m_{\mathbf{k}}^{\mathrm{X}}\left(B_{\mathbf{p}_{\|}}^{\dagger} B_{\mathbf{p}_{\|}-\mathbf{k}} e^{-i \Omega_{\mathbf{k}}^{\mathrm{ac} t} t}-B_{\mathbf{p}_{\|}-\mathbf{k}}^{\dagger} B_{\mathbf{p}_{\|}} e^{i \Omega_{\mathbf{k}}^{\mathrm{ac}} t}\right)\right]
\end{aligned}
$$

where $\mathbf{p}_{\|}$is the in-plane wavevector, $B_{\mathbf{p}_{\|}}$are $\alpha_{\mathbf{p}_{\|}}$ are the QW exciton and MC photon operators, respectively, $\hbar \omega_{\mathbf{p}_{\|}}^{\mathrm{X}}=\hbar \omega_{t}+\left(\hbar^{2} p_{\|}^{2}\right) /\left(2 M_{x}\right)$ and $\hbar \omega_{\mathbf{p}_{\|}}^{\mathrm{MC}}=$ $\hbar \sqrt{\omega_{0}^{2}+c^{2} p_{\|}^{2} / \varepsilon_{b}}$ are the corresponding energies, $M_{x}$ is the QW exciton translational mass, $\omega_{0}$ is the cavity (photon) eigenfrequency, and $\varepsilon_{b}$ is the background dielectric constant. The oscillator strength of the resonant coupling between QW excitons and MC photons is characterized by the polariton Rabi frequency, $\Omega_{\mathrm{X}}^{\mathrm{MC}}$. The parametric interaction of MC polaritons with the acoustic pump wave occurs through their excitonic component and is given by $m_{\mathbf{k}}^{\mathrm{X}}=m_{\mathrm{x}-\mathrm{ac}} \sqrt{N_{0}^{\mathrm{ph}}}$. Here $m_{\mathrm{x}-\mathrm{ac}}$ is the matrix element of exciton-phonon interaction and $N_{0}^{\mathrm{ph}} \propto I_{\mathrm{ac}}$ is the concentration of the coherent acoustic phonons. For definiteness we assume a ( $\mathrm{GaAs}) \lambda$-microcavity so that $\omega_{0}=(2 \pi c) /\left(L_{z} \sqrt{\varepsilon_{b}}\right)$, where $L_{z}$ is the MC thickness.

As we discuss in detail below, the SAW properties and the SAW generation technique are very suitable for the effective realization of the resonant acousto-optic effect in optical microcavities. The interaction of QW excitons with SAWs is piezoelectric so that the matrix element $m_{\mathbf{k}}^{\mathrm{X}}$ is given by

$$
m_{\mathbf{k}}^{\mathrm{X}}=\left(\frac{2 N_{0}^{\mathrm{ph}} \hbar k}{\rho v_{s}}\right)^{1 / 2} \frac{\pi e}{\varepsilon_{b}} \mathrm{e}_{14} k a_{\mathrm{B}}^{2}\left(\frac{m_{\mathrm{e}}-m_{\mathrm{h}}}{M_{x}}\right),
$$


where $\rho$ is the mass density, $v_{s} \equiv v_{\mathrm{SAW}}$ is the sound velocity, $\mathrm{e}_{14}$ is the relevant component of an electromechanical tensor of the electron - phonon piezoelectric interaction, $a_{\mathrm{B}}$ is the $\mathrm{QW}$ exciton Bohr radius, and $m_{\mathrm{e}(\mathrm{h})}$ is the QW electron (hole) mass. Equation (2) is valid for $\left(a_{\mathrm{B}} k\right) / 2 \ll 1$. For GaAs-based structures this means that the wavevector $k$ of the SAW should be less than $5 \times 10^{5} \mathrm{~cm}^{-1}$. For GaAs one has $\mathrm{e}_{14}=$ $0.48 \times 10^{5} \mathrm{~g}^{1 / 2} \mathrm{~s}^{-1} \mathrm{~cm}^{-1 / 2}[35]$ and $v_{\mathrm{SAW}} \simeq 2.87 \times 10^{5} \mathrm{~cm} / \mathrm{s}$ [32]. The latter value clearly shows that the SAW velocity is less than the sound velocities of bulk, transverse and longitudinal, acoustic phonons. The concentration of the coherent phonons $N_{0}^{\mathrm{ph}}$, associated with the SAW, is given by $N_{0}^{\mathrm{ph}}=I_{\mathrm{ac}} /\left(v_{s} \hbar \Omega_{\mathbf{k}}^{\mathrm{ac}}\right)$. Here the phonon eigenfrequency $\Omega_{\mathbf{k}}^{\text {ac }}=v_{s} k$ is resonant to the angular SAW frequency $\Omega_{\mathrm{SAW}}$, i.e. $\Omega_{\mathbf{k}}^{\mathrm{ac}}=\Omega_{\mathrm{SAW}}$. Usually the SAW frequency is given in terms of $\nu_{\mathrm{ac}} \equiv \nu_{\mathrm{SAW}}=\Omega_{\mathrm{SAW}} /(2 \pi)$ so that the phonon wavelength is $\lambda_{\mathrm{ac}} \equiv \lambda_{\mathrm{SAW}}=v_{\mathrm{SAW}} / \nu_{\mathrm{SAW}}$.

The explicit time dependence of the Hamiltonian (1), due to the cw acoustic pump wave, can be removed by the canonical transformation:

$$
\begin{aligned}
& S=\exp \left[i t \sum_{\mathbf{p}_{\|}}\left(\mathbf{v}_{\mathbf{s}} \cdot \mathbf{p}_{\|}\right)\left(B_{\mathbf{p}_{\|}}^{\dagger} B_{\mathbf{p}_{\|}}+\alpha_{\mathbf{p}_{\|}}^{\dagger} \alpha_{\mathbf{p}_{\|}}\right)\right] \\
& B_{\mathbf{p}_{\|}} \rightarrow S B_{\mathbf{p}_{\|}} S^{\dagger}=B_{\mathbf{p}_{\|}} e^{-i\left(\mathbf{v}_{\mathbf{s}} \cdot \mathbf{p}_{\|}\right) t} \\
& \alpha_{\mathbf{p}_{\|}} \rightarrow S \alpha_{\mathbf{p}_{\|}} S^{\dagger}=\alpha_{\mathbf{p}_{\|}} e^{-i\left(\mathbf{v}_{\mathbf{s}} \cdot \mathbf{p}_{\|}\right) t}
\end{aligned}
$$

where $\mathbf{v}_{\mathbf{s}}=v_{s}(\mathbf{k} / k)$ and, therefore, $\left(\mathbf{v}_{\mathbf{s}} \cdot \mathbf{p}_{\|}\right)=\Omega_{\mathbf{k}}^{\text {ac }}[(\mathbf{k}$. $\left.\mathbf{p}_{\|}\right) / k^{2}$. The canonical transformation (3) means the use of the coordinate system which moves with the acoustic wave. In this case the quadratic Hamiltonian $\tilde{H}=$ $S H S^{\dagger}-i S\left(\partial S^{\dagger} / \partial t\right)$ is time-independent at least insofar as the rotating wave approximation made in equation (1) is respected. The Hamiltonian $\tilde{H}$ is then exactly-solvable. Its diagonalization yields the dispersion equation for $\mathrm{MC}$ polaritons parametrically driven by the coherent (surface) acoustic wave:

$$
\begin{gathered}
\omega^{2}-\left(\tilde{\omega}_{\mathbf{p}_{\|}}^{\mathrm{X}}\right)^{2}-\frac{\left(\omega \Omega_{\mathrm{X}}^{\mathrm{MC}}\right)^{2}}{\omega^{2}-\left(\tilde{\omega}_{\mathbf{p}_{\|} \mathrm{MC}}^{\mathrm{M}}\right)^{2}} \\
-\frac{4 \omega_{t}^{2}\left|m_{\mathbf{k}}^{\mathrm{X}}\right|^{2}}{\omega^{2}-\left(\tilde{\omega}_{\mathbf{p}_{\|}+\mathbf{k}}^{\mathrm{X}}\right)^{2}-\frac{\left(\omega \Omega_{\mathrm{M}}^{\mathrm{MC}}\right)^{2}}{\omega^{2}-\left(\tilde{\omega}_{\mathbf{p}_{\|}+\mathbf{k}}^{\mathrm{MC}}\right)^{2}}-M_{\mathbf{p}_{\|}+2 \mathbf{k}}} \\
-\frac{4 \omega_{t}^{2}\left|m_{\mathbf{k}}^{\mathrm{X}}\right|^{2}}{\omega^{2}-\left(\tilde{\omega}_{\mathbf{p}_{\|}-\mathbf{k}}^{\mathrm{X}}\right)^{2}-\frac{\left(\omega \Omega_{\mathrm{X}}^{\mathrm{MC}}\right)^{2}}{\omega^{2}-\left(\widetilde{\omega}_{\mathbf{p}_{\|}-\mathbf{k}}^{\mathrm{MC}}\right)^{2}}-M_{\mathbf{p}_{\|}-2 \mathbf{k}}}=0,
\end{gathered}
$$

where $M_{\mathbf{p}_{\|} \pm 2 \mathbf{k}}$ is given through $M_{\mathbf{p}_{\|} \pm 3 \mathbf{k}}, M_{\mathbf{p}_{\|} \pm 3 \mathbf{k}}$ through $M_{\mathbf{p}_{\|} \pm 4 \mathbf{k}}$, etc. by the recurrent formula valid for a positive integer $n \geq 1$ :

$$
M_{\mathbf{p}_{\|} \pm n \mathbf{k}}=\frac{4 \omega_{t}^{2}\left|m_{\mathbf{k}}^{\mathrm{X}}\right|^{2}}{\omega^{2}-\left(\tilde{\omega}_{\mathbf{p}_{\|} \pm n \mathbf{k}}^{\mathrm{X}}\right)^{2}-\frac{\left(\omega \Omega_{\mathrm{X}}^{\mathrm{MC} C}\right)^{2}}{\omega^{2}-\left(\tilde{\omega}_{\mathbf{p}_{\|} \pm n \mathbf{k}}^{\mathrm{MC}}\right)^{2}}-M_{\mathbf{p}_{\|} \pm(n+1) \mathbf{k}}}
$$

and $\hbar \tilde{\omega}_{\mathbf{p}_{\|} \pm n \mathbf{k}}^{\mathrm{X}, \mathrm{MC}}=\hbar \omega_{\mathbf{p}_{\|} \pm n \mathbf{k}}^{\mathrm{X}, \mathrm{MC}} \mp n \hbar \Omega_{\mathbf{k}}^{\mathrm{ac}}=\hbar \omega_{\mathbf{p}_{\|} \pm n \mathbf{k}}^{\mathrm{X}, \mathrm{MC}} \mp n \hbar\left(\mathbf{v}_{\mathbf{s}}\right.$. $\mathbf{k}$ ) are the $\mathrm{QW}$ exciton and $\mathrm{MC}$ photon quasi-energies, respectively.

In the derivation of the dispersion equation (4) we have treated the interaction between $\mathrm{QW}$ excitons and $\mathrm{MC}$ photons in its general, nonresonant approximation. If $\left|m_{\mathbf{k}}^{\mathrm{X}}\right|^{2} \propto I_{\mathrm{ac}}$ is equal to zero, i.e. in the absence of the acoustic pump wave, equation (4) reduces to the MC polariton dispersion relationship:

$$
\frac{c^{2} p_{\|}^{2}}{\varepsilon_{b}}+\omega_{0}^{2}=\omega^{2}+\frac{\omega^{2}\left(\Omega_{\mathrm{X}}^{\mathrm{MC}}\right)^{2}}{\omega_{t}^{2}+\hbar \omega_{t} p_{\|}^{2} / M_{x}-\omega^{2}} .
$$

In this case the exciton and photon components of MC polaritons, $\varphi^{\mathrm{MC}}$ and $\psi^{\mathrm{MC}}$, are given by

$$
\begin{aligned}
& \varphi^{\mathrm{MC}}\left(p_{\|}\right)=\frac{\left(\Omega_{\mathrm{X}}^{\mathrm{MC}}\right)^{2}}{\left(\Omega_{\mathrm{X}}^{\mathrm{MC}}\right)^{2}+4\left[\omega_{t}+\hbar p_{\|}^{2} /\left(2 M_{x}\right)-\omega_{\mathrm{pol}}^{\mathrm{MC}}\left(p_{\|}\right)\right]^{2}}, \\
& \psi^{\mathrm{MC}}\left(p_{\|}\right)=1-\varphi^{\mathrm{MC}}\left(p_{\|}\right),
\end{aligned}
$$

respectively, where $\omega_{\text {pol }}^{\mathrm{MC}}=\omega_{\mathrm{LPB}(\mathrm{UPB})}^{\mathrm{MC}}\left(p_{\|}\right)$is the $\mathrm{MC}$ lower (upper) dispersion branch determined by equation (6).

The dispersion equation (4) describes the resonant acousto-optic (Stark) effect for MC polaritons. The equation is suitable for numerical calculations within a truncated scheme, i.e. by putting $M_{\mathbf{p}_{\|} \pm n \mathbf{k}}$ equal to zero at some integer $n=n^{\max }>1$. The truncated scheme quickly converges with increasing $n^{\max }$. According to equation (4), with increasing $I_{\mathrm{ac}}$ the optical band gaps (optical stop gaps) open up and develop in the $\mathrm{MC}$ polariton spectrum. The gaps arise for the MC polariton states resonantly coupled by one-, two-, etc. phonon-assisted transitions induced by the acoustic pump wave. The quasi-energy spectrum (4) can be interpreted in terms of the Brillouin energy bands: the initial MC polariton dispersion is modulated by the periodic potential associated with the coherent acoustic wave. The latter potential is periodic not only in space, with the "lattice" constant $\lambda_{\mathrm{ac}}$, but also in time, with the period $1 / \nu_{\mathrm{ac}}$. This gives rise to the quasi-energies $\hbar \tilde{\omega}_{\mathbf{p}_{\|} \pm n \mathbf{k}}^{\mathrm{X}, \mathrm{MC}}$ in equation (4) (the concept of quasi-energies has been introduced and developed in [36]). Thus not only two in-plane momenta, $\hbar \mathbf{p}_{\|}$ and $\hbar \mathbf{p}_{\|} \pm n \hbar \mathbf{k}$, are equivalent for acoustically-driven MC polaritons, but in a similar way two energies, $\hbar \omega_{\mathbf{p} \|}^{\mathrm{X}, \mathrm{MC}}$ and $\hbar \omega_{\mathbf{p}_{\|} \pm n \mathbf{k}}^{\mathrm{X}, \mathrm{MC}} \mp n \hbar \Omega_{\mathbf{k}}^{\mathrm{ac}}$, are also indistinguishable.

The quasi-energy spectrum of bulk polaritons driven by a bulk acousic pump wave has been discussed in detail in [4]. Here we concentrate on the first (main) optical stop gap associated with the one-phonon resonant transition between the MC polariton states. In figure 1(a) we plot the initial MC polariton dispersion calculated by equation (6) for zero detuning at $p_{\|}=0$ between the cavity mode and the QW exciton energy, i.e. for $\omega_{0}=\omega_{t}$. The parameters relevant to GaAs-based microcavities have been used in the calculations: the Rabi energy $\hbar \Omega_{\mathrm{X}}^{\mathrm{MC}}=3.7 \mathrm{meV}$, the dielectric constant $\varepsilon_{b}=12.3$ 
and the QW exciton energy $E_{\mathrm{X}}=\hbar \omega_{t}=1.522 \mathrm{eV}$. The applied SAW is characterized by the wavevector $k=5 \times 10^{4} \mathrm{~cm}^{-1}$ and the frequency $\nu_{\mathrm{ac}}=2.28 \mathrm{GHz}$ (see figure 1(a), where only the one-phonon-assisted transition within the lower MC polariton dispersion branch is indicated by the arrow 1LPLP). Figure 1(b) illustrates how the first optical band gap develops with increasing acoustic intensity $I_{\mathrm{ac}} \equiv I_{\mathrm{SAW}}$. The plot has been calculated by equation (4) using the truncated scheme with $n^{\max }=4$.

The first (main) optical band gap is due to the MC polariton states $\left\{\mathbf{p}_{\|}, \omega_{\mathrm{LPB}}^{\mathrm{MC}}\left(\mathbf{p}_{\|}\right)\right\}$and $\left\{\mathbf{p}_{\|}-\mathbf{k}, \omega_{\mathrm{LPB}}^{\mathrm{MC}}\left(\mathbf{p}_{\|}-\mathbf{k}\right)\right\}$ resonantly coupled by the one-phonon-assisted transition induced by the SAW $\left\{\mathbf{k}, \Omega_{\mathbf{k}}^{\mathrm{ac}} \equiv \Omega_{\mathrm{SAW}}\right\}$ (see figures 1(a) and 1(b)). Because the energy of a SAW phonon is rather small, $\hbar \Omega_{\mathrm{SAW}} \simeq 9.4 \mu \mathrm{eV}$, for the one-dimensional geometry, $\mathbf{p}_{\|} \| \mathbf{k}$, one has that the momentum-energy matching condition is nearly satisfied for the MC states $\left\{\mathbf{p}_{\|}, \omega_{\mathrm{LPB}}^{\mathrm{MC}}\left(\mathbf{p}_{\|}\right)\right\}=\left\{\mathbf{k} / 2, \omega_{\mathrm{LPB}}^{\mathrm{MC}}(k / 2)\right\}$ and $\left\{\mathbf{p}_{\|}-\mathbf{k}, \omega_{\mathrm{LPB}}^{\mathrm{MC}}\left(\mathbf{p}_{\|}-\mathbf{k}\right)\right\}=\left\{-\mathbf{k} / 2, \omega_{\mathrm{LPB}}^{\mathrm{MC}}(k / 2)\right\}$. Thus, using equation (4), we conclude that the first optical band gap $\Delta_{\mathrm{ac}}^{\mathrm{MC}} \equiv \Delta_{\mathrm{ac}}^{\mathrm{MC}(n=1)} \propto \sqrt{I_{\mathrm{ac}}}$ is given approximately by

$$
\begin{aligned}
\Delta_{\mathrm{ac}}^{\mathrm{MC}} & =\Delta_{\mathrm{ac}}^{\mathrm{X}}\left(I_{\mathrm{ac}}\right)\left[\varphi^{\mathrm{MC}}(\mathbf{k} / 2) \varphi^{\mathrm{MC}}(-\mathbf{k} / 2)\right]^{1 / 2} \\
& \equiv \Delta_{\mathrm{ac}}^{\mathrm{X}}\left(I_{\mathrm{ac}}\right) \varphi^{\mathrm{MC}}(k / 2),
\end{aligned}
$$

where $\Delta_{\mathrm{ac}}^{\mathrm{X}}=2\left|m_{\mathrm{k}}^{\mathrm{X}}\right| \propto \sqrt{I_{\mathrm{ac}}}$ is the stop band for opticallyundressed QW excitons, and $\varphi^{\mathrm{MC}}=\varphi_{\mathrm{LPB}}^{\mathrm{MC}}$ is given by equation (7). For the piezoelectric interaction, which is determined by equation (2), one has that $\Delta_{\mathrm{ac}}^{\mathrm{X}}$ is proportional to the phonon wavevector $k$. According to equation (8), the acoustically created gap in the MC polariton spectrum can also be interpreted in terms of coherent phonon-mediated coupling of virtual, opticallydressed QW excitons with the in-plane wavevectors $\mathbf{k} / 2$ and $-\mathbf{k} / 2$. In the time domain, the optical band gap can be visualized as acoustically induced coherent oscillations of MC polaritons, forth and back between the states $\mathbf{k} / 2$ and $-\mathbf{k} / 2$. The frequency of the oscillations is given by $\Delta_{\mathrm{ac}}^{\mathrm{MC}}$.

An acoustic intensity threshold for the resonant acousto-optic Stark effect is approximately given by the condition $\Delta_{\mathrm{ac}}^{\mathrm{MC}}\left(I_{\mathrm{ac}}\right) \geq \operatorname{Max}\left\{\Gamma_{\mathrm{R}} / 2, \gamma_{\mathrm{X}} / 2\right\}$, where $\Gamma_{\mathrm{R}}$ is the inverse radiative lifetime of $\mathrm{MC}$ photons and $\gamma_{\mathrm{X}}$ is the rate of incoherent scattering of $\mathrm{QW}$ excitons. The rates have to be included in the dispersion equation (4) by the substitution $\omega_{t}^{2} \rightarrow \omega_{t}^{2}-i \omega \gamma_{\mathrm{X}}$ and $\omega_{0}^{2} \rightarrow \omega_{0}^{2}-i \omega \Gamma_{\mathrm{R}}$. Note that both damping constants, $\Gamma_{\mathrm{R}}$ and $\gamma_{\mathrm{X}}$, refer to the decay rates of the occupation numbers of the relevant in-plane $\mathrm{MC}$ photon and $\mathrm{QW}$ exciton modes. The corresponding $T_{1}$-times are given by $T_{1}^{\mathrm{R}}=\hbar / \Gamma_{\mathrm{R}}$ and $T_{1}^{\mathrm{X}}=\hbar / \gamma_{\mathrm{X}}$, respectively, and we assume that the $T_{2}=2 T_{1}$ limit holds for MC photons and QW excitons. A more precise condition for the threshold $I_{\mathrm{ac}}$ is

$$
\Delta_{\mathrm{ac}}^{\mathrm{MC}}\left(I_{\mathrm{ac}}, \mathbf{k}\right) \geq \frac{1}{2} \operatorname{Max}\left\{\begin{array}{l}
\psi^{\mathrm{MC}}(k / 2) \Gamma_{\mathrm{R}}, \\
\varphi^{\mathrm{MC}}(k / 2) \gamma_{\mathrm{X}},
\end{array}\right.
$$

where $\psi^{\mathrm{MC}}$ and $\varphi^{\mathrm{MC}}$ are given by equation (7). Using equation (8), the second inequality can also be re-written as $\Delta_{\mathrm{ac}}^{\mathrm{X}} \geq \gamma_{\mathrm{X}} / 2$. For the up-to-date GaAs-based microcavites at helium temperatures, $\Gamma_{R}$ is much larger than $\gamma_{\mathrm{X}}$. For example, for high-quality GaAs MCs the radiative lifetime of MC photons $T_{1}^{\mathrm{R}}$ is about $2-3 \mathrm{ps}$ [12] so that $\hbar \Gamma_{\mathrm{R}} \simeq 0.3 \mathrm{meV}$. In the meantime, at $T=4.2 \mathrm{~K}$ the scattering rate of QW excitons yields $\hbar \gamma_{\mathrm{X}} \simeq 30-60 \mu \mathrm{eV}$ $[37,38]$. The latter values refer to the low density limit of QW excitons. Note that we do have this limit for the resonant acousto-optic Stark effect: our scheme does not require high optical excitations, while the acoustic pump wave cannot generate the QW excitons itself in intrinsic, undoped nano-structures. In contrast, the conventional optical Stark effect, mediated by excitons, needs a highintensity electromagnetic wave which nearly resonates with the interband electronic transitions and, therefore, inevitably populates the excitonic and free electron (hole) states. As a result, $\gamma_{\mathrm{X}}$ considerably increases in an uncontrolled way and relaxes the optical Stark effect.

From the dispersion equation (4) we find the acoustically-mediated excitonic susceptibility for MC polaritons:

$$
\begin{aligned}
\chi_{\mathrm{x}-\mathrm{ac}}^{\mathrm{MC}}\left(I_{\mathrm{ac}}\right) & =-\frac{\varepsilon_{b}}{4 \pi} \frac{\left(\Omega_{\mathrm{X}}^{\mathrm{MC}}\right)^{2}}{\omega^{2}-\left(\tilde{\omega}_{\mathbf{p}_{\|}}^{\mathrm{X}}\right)^{2}} \\
& \times \frac{M_{\mathbf{p}_{\|}+\mathbf{k}}+M_{\mathbf{p}_{\|}-\mathbf{k}}}{\omega^{2}-\left(\tilde{\omega}_{\mathbf{p}_{\|}}^{\mathrm{X}}\right)^{2}-M_{\mathbf{p}_{\|}+\mathbf{k}}-M_{\mathbf{p}_{\|}-\mathbf{k}}},
\end{aligned}
$$

where $M_{\mathbf{p}_{\|} \pm \mathbf{k}}$ is given by equation (5) with $n=1$. The susceptibility $\chi_{\mathrm{x}-\mathrm{ac}}^{\mathrm{MC}}$, which is linear with respect to the light field, can also be interpreted as a resonant acousto-optical nonlinear susceptibility $\chi_{\mathrm{x}-\mathrm{ac}}^{\mathrm{MC}}\left(I_{\mathrm{ac}}\right)=$ $\sum_{n=1}^{\infty} \chi_{\mathrm{x}-\mathrm{ac}}^{(2 n+1)} I_{\mathrm{ac}}^{n}$. Note that $\chi_{\mathrm{x}-\mathrm{ac}}^{\mathrm{MC}}$ is strongly enhanced due to small detuning $\omega-\omega_{t}$ from the QW excitonic resonance and due to the small values of $\Gamma_{\mathrm{R}}$ and $\gamma_{\mathrm{X}}$. According to equation (10), the acoustically-induced change of the dielectric constant $\varepsilon_{b}$, associated with the third-order $\chi_{\mathrm{x}-\mathrm{ac}}^{(3)}$, has a maximum value given by

$$
\begin{aligned}
\left.\frac{\left|\delta \varepsilon_{b}^{(3)}\left(\omega, I_{\mathrm{ac}}\right)\right|}{\varepsilon_{b}}\right|_{\max } & =\frac{\left(\Omega_{\mathrm{X}}^{\mathrm{MC}}\right)^{2} \varphi^{\mathrm{MC}}}{2 \omega_{t}\left(\varphi^{\mathrm{MC}} \gamma_{\mathrm{X}}+\psi^{\mathrm{MC}} \Gamma_{\mathrm{R}}\right)} \\
& \times \frac{\left(\Delta_{\mathrm{ac}}^{\mathrm{X}}\right)^{2}}{\left(\omega-\omega_{t}\right)^{2}} \propto I_{\mathrm{ac}},
\end{aligned}
$$

where the light field of frequency $\omega$ resonates with the centre of the acoustically-induced band gap, and we assume that $\left|\omega-\omega_{t}\right| \gg \gamma_{\mathrm{X}} / 2$. For example, for the parameters relevant to figure 1 (b) one has $\omega-\omega_{t} \simeq-0.49 \mathrm{meV}$, $\varphi_{\mathrm{LPB}}^{\mathrm{MC}}(\omega) \simeq 0.93$ and $\psi_{\mathrm{LPB}}^{\mathrm{MC}}(\omega) \simeq 0.07$ so that equation (11) yields $\left|\delta \varepsilon_{b}^{(3)}\left(I_{\mathrm{SAW}}=4.5 \mathrm{~mW} / \mathrm{mm}\right)\right|_{\max } \simeq 0.54$. The latter value clearly shows that the resonant acoustooptics of MC polaritons deals with the giant acoustooptical nonlinearities. 


\section{DISCUSSION}

If the incoherent scattering processes of QW excitons and the radiative decay rates of $\mathrm{MC}$ polaritons are neglected, the acoustically-induced stop gaps in the MC polariton spectrum correspond to a real and negative effective dielectric constant $\varepsilon^{\text {eff }}=\varepsilon^{\text {eff }}\left(\omega, I_{\mathrm{ac}}\right)$ and yield total reflection for the weak light field which activates and probes the acoustically-driven MC polaritons. In this case sharp spikes, which are associated with the acoustically-induced optical stop gaps, arise and develop with increasing $I_{\mathrm{ac}}$ in the reflection spectrum of acoustically-dressed MC polaritons (the reflection spectrum of acoustically-driven excitons in bulk GaAs is shown in [4]). The main acoustically-induced spike corresponds to the one-phonon resonant coupling between two MC polariton states (see figures 1(a) and 1(b)). The contrast of the spikes decreases with the increasing scattering rates $\gamma_{\mathrm{X}}$ and $\Gamma_{\mathrm{R}}$, and finally the acoustically-induced changes of the reflection spectrum disappears when $\Delta_{\mathrm{ac}}^{\mathrm{MC}}=\Delta_{\mathrm{ac}}^{\mathrm{MC}}\left(I_{\mathrm{ac}}\right)$ becomes much less than $\operatorname{Max}\left\{\Gamma_{\mathrm{R}} / 2, \gamma_{\mathrm{X}} / 2\right\}$. Note that the spectral position of the acoustically-induced band gaps can easily be tuned by sub-GHz changes in the frequency $\Omega_{\mathbf{k}}^{\mathrm{ac}}$ of the acoustic pump wave.

The standard acousto-optic effect can be interpreted in terms of optical diffraction of the incident light field by the acoustically-induced grating of the period $\lambda_{\mathrm{ac}}$. In this case a coherent acoustic pump wave can be treated as nonpropagating, because $c \gg v_{s}$. The acousticallyinduced diffraction grating is due to the photoelastic effect, which gives rise to a small, nonresonant change of the dielectric constant, $\left|\delta \varepsilon_{b}\right| \ll \varepsilon_{b}$, at acoustic wave peaks (pressure maxima so that usually $\delta \varepsilon_{b}$ is positive) and troughs (pressure minima so that usually $\delta \varepsilon_{b}$ is negative). In sharp contrast with the above picture, the resonant acousto-optic effect we describe deals with a quantum diffraction of optically-active MC excitons by an ultrasonic coherent wave. In this case the interaction of two "matter" waves, the excitonic polarization and the acoustic field, is much more effective than nonresonant coupling of photons with acoustic phonons. In the meantime the photon component of optically-dressed MC excitons, i.e. of MC polaritons, can be large enough to ensure an efficient resonant conversion "MC photon $\leftrightarrow \mathrm{MC}$ exciton". This explains qualitatively the origin of the giant acousto-optic nonlinearities $4 \pi \chi_{\mathrm{x}-\mathrm{ac}}^{\mathrm{MC}} \gg \delta \varepsilon_{b}$, which are mediated by the exciton states nearly resonant with the acoustic and optic fields simultaneously.

For the frequency band of visible light the onedimensional geometry of the acousto-optic interaction can hardly be realized within the nonresonant acoustooptic effect in bulk materials, because in order to couple two mutually-opposite states of the photon cone, $\mathbf{p}$ and $\mathbf{p}-\mathbf{k} \simeq-\mathbf{p}$, one needs an acoustic frequency $\nu_{\mathrm{ac}} \gtrsim 20 \mathrm{GHz}$. The up-to-date SAW technique deals with $\nu_{\mathrm{ac}} \lesssim 10 \mathrm{GHz}$. Thus usually the photon wavevector $|\mathbf{p}|$ is much larger than $|\mathbf{k}|=2 \pi / \lambda_{\text {ac }}$, so that only a nearly normal interaction geometry, $\mathbf{p} \perp \mathbf{k}$, is used in the standard Raman-Nath (transmission type) and Bragg (reflection type) acousto-optic diffraction grating schemes applied to a bulk medium [2,39]. The $1 \mathrm{D}$ interaction geometry can easily be realized for the resonant acousto-optic effect in optical microcavities. Namely, due to a particular shape of the MC polariton dispersion branches with the closely separated absolute energy minima at $\mathbf{p}_{\|}=\mathbf{0}$, a pump ultrasonic acoustic wave of any frequency $\nu_{\text {ac }} \lesssim 2-3 \mathrm{GHz}$ couples two counter-propagating MC polaritons with wavevectors $\mathbf{p}_{\|} \simeq \pm \mathbf{k} / 2$ (see figure $1(\mathrm{a})$ ). By the same reason the $1 \mathrm{D}$ geometry of the phononmediated interaction between counter-propagating photons can be realized by using the nonresonant acoustooptical nonlinearities in SAW-driven optical fibers.

Due to the small values of the nonresonant acoustooptical nonlinearities, the interaction length between the photon and phonon fields, $l_{\gamma-\text { ac }}$, needed for the usual acousto-optic effect, is large. For example, for the SAWdriven optical fibers, $l_{\gamma-\text { ac }}$ is on a scale of tens of centimeters $[40,41]$. In sharp contrast with this, the resonant acousto-optic Stark effect for MC polaritons requires the interaction length $l_{\mathrm{MC}-\mathrm{ac}} \sim 10 \mu \mathrm{m}$, i.e. by four orders of magnitude less than that in the traditional acoustooptics. By using the dispersion equation (4) we derive the following estimate for $l_{\mathrm{MC}-\mathrm{ac}}$ associated with the main acoustically-induced stop gap (one-phonon transition):

$$
l_{\mathrm{MC}-\mathrm{ac}} \simeq \frac{4 \pi v_{\mathrm{pol}}^{\mathrm{MC}}(k / 2)}{\Delta_{\mathrm{ac}}^{\mathrm{MC}}\left(I_{\mathrm{ac}}, k / 2\right)} \propto \frac{1}{I_{\mathrm{ac}}},
$$

where $\Delta_{\mathrm{ac}}^{\mathrm{MC}}$ is given by equation (8) and $v_{\text {pol }}^{\mathrm{MC}}\left(p_{\|}\right)=$ $\partial \omega_{\text {pol }}^{\mathrm{MC}} / \partial p_{\|}$is the $\mathrm{MC}$ polariton group velocity. For $I_{\mathrm{SAW}}=4.5 \mathrm{~mW} / \mathrm{mm}$ and $\nu_{\mathrm{ac}}=2.28 \mathrm{GHz}$, equation $(12)$ yields $l_{\mathrm{MC}-\mathrm{ac}} \simeq 13.3 \mu \mathrm{m}$ for a zero-detuning GaAs-based microcavity with the Rabi energy given by $\hbar \Omega_{\mathrm{X}}^{\mathrm{MC}}=$ $3.7 \mathrm{meV}$ (see figure 1(b)). For the above values of $\nu_{\mathrm{ac}}$ and $\hbar \Omega_{\mathrm{X}}^{\mathrm{MC}}$ the relevant polariton group velocity is $v_{\text {pol }}^{\mathrm{MC}}(k / 2) \simeq 0.53 \times 10^{8} \mathrm{~cm} / \mathrm{s}$.

Two schemes "SAW pumping - optical probing" can be proposed for semiconductor microcavities: one deals with the use of bulk incoming/outgoing photons, which resonantly couple with MC polaritons through a MC Bragg reflector, and another involves in-plane light delivered to and collected from the lateral surfaces of a MC chip (see figure 2). The evanescent acoustic field associated with a SAW decays nearly exponentially in the $z$-direction (the direction normal to the surface and, therefore, to the phonon wavevector $\mathbf{k}$ ) with a characteristic decay length $l_{\text {SAW }}^{z}$ of a few $\lambda_{\text {ac }}$. Note that for acoustically pumped GaAs-based microstructures the SAWs can usually be interpreted in terms of a Rayleigh wave $[42,43]$. For the SAW frequency $\nu_{\mathrm{SAW}}=2.28 \mathrm{GHz}$, which has been used to calculate the plots shown in figures 1(a) and 1 (b), the phonon wavevector is $k=5 \times 10^{4} \mathrm{~cm}^{-1}$ so that 
$\lambda_{\mathrm{ac}} \simeq 1.3 \mu \mathrm{m}$. In this case $l_{\mathrm{SAW}}^{z}<l_{\mathrm{MC}-\mathrm{ac}}$ and, therefore, the first scheme for the realization of the SAW-induced resonant acousto-optic effect is less favourable than the second one.

The polariton effect in GaN-, ZnSe- and CdTe-based microcavities can be large, $\hbar \Omega_{\mathrm{X}}^{\mathrm{MC}} \gtrsim 20-30 \mathrm{meV}$ $[8,10,14,44]$. A self-consistent combination of the large binding energies of $\mathrm{QW}$ excitons and the large $\mathrm{MC}$ Rabi-splitting energies gives rise to the well-defined inplane polariton eigenstates in the above microstructures even at the room temperature [14]. Thus the resonant acousto-optic Stark effect can also be realized in these structures at high temperatures. At the room temperature, however, the rate of incoherent scattering of $\mathrm{QW}$ excitons is larger than the radiative width of MC polaritons, i.e. $\gamma_{X}>\Gamma_{R}$. In this case the acoustic intensity threshold of the Stark effect is determined by $\hbar \Delta_{\mathrm{ac}}^{\mathrm{MC}}\left(I_{\mathrm{ac}}\right) \gtrsim \hbar \gamma_{\mathrm{X}} / 2 \sim 1 \mathrm{meV}$.

The "optical pumping - optical probing" scheme mediated by acoustic phonons, i.e. the phonon-mediated optical Stark effect for QW excitons in microcavities, deals with the same three-particle exciton-phonon interaction as in the resonant acousto-optic effect. Therefore, a similar energy scale for the pump-induced spectral changes in both effects requires nearly the same concentrations of coherent virtual excitons $N_{\mathrm{X}}^{\mathrm{MC}}=$ $\left[\varphi^{\mathrm{MC}}(\tilde{\omega}) I_{\mathrm{opt}}\right] /\left[v_{\mathrm{pol}}^{\mathrm{MC}}(\tilde{\omega}) \hbar \tilde{\omega}\right]$ driven by the light field of intensity $I_{\mathrm{opt}}$ in the first case and coherent acoustic phonons $N_{0}^{\mathrm{ph}}=I_{\mathrm{ac}} /\left(v_{s} \hbar \Omega_{\mathbf{k}}^{\mathrm{ac}}\right)$ used in the latter one. Here $\tilde{\omega} \simeq \omega_{0}$ is the frequency of the pump optical wave, which induces a high-intensity MC polariton, and the excitonic component $\varphi^{\mathrm{MC}}(\tilde{\omega})$ of the pump polariton is given by equation (7). Thus in order to generate $N_{\mathrm{X}}^{\mathrm{MC}} \sim N_{0}^{\mathrm{ph}}$ one needs much higher optical intensities than those necessary for the resonant acousto-optic Stark effect, i.e. $I_{\mathrm{opt}} \gg I_{\mathrm{ac}}$, because the $\mathrm{MC}$ polaritons have much higher frequency and lager group velocity than the acoustic phonons $\left(\tilde{\omega} \gg \Omega_{\mathbf{k}}^{\mathrm{ac}}\right.$ and $\left.v_{\mathrm{pol}}^{\mathrm{MC}}>v_{s}\right)$. For example, the intensity $I_{\mathrm{SAW}}=4.5 \mathrm{~mW} / \mathrm{mm}$, which gives rise to the acoustically-induced spectrum plotted in figure 1(b), corresponds to the average concentration of the coherent phonons, distributed in the surface layer of thickness $\lambda_{\text {ac }},\left\langle N_{0}^{\mathrm{ph}}\right\rangle \simeq 0.92 \times 10^{22} \mathrm{~cm}^{-3}$. A similar concentration of QW excitons definitely cannot be realized in GaAs nano-structures, due to complete removal of the excitonic states by the phase space filling effect.

\section{ACOUSTICALLY-DRIVEN MICROCAVITIES FOR OPTICAL MODULATION AND SWITCHING}

For GaAs-based microcavities, the thickness of a top MC Bragg reflector is usually about $2-3 \mu \mathrm{m}$, i.e. is comparable with the SAW decay length $l_{\mathrm{SAW}}^{z}$ in the $z$-direction. In the meantime the in-plane interaction length $l_{\mathrm{MC}-\mathrm{ac}}$ is compatible with the length scale needed for the effective work of an interdigital transducer (IT, a grid of equally-separated metalic stripes deposited on the surface of a piezoelectric crystal; an AC voltage applied to the IT induces a SAW). This makes the resonant acousto-optic effect in SAW-driven microcavities very attractive for possible applications [5]. In figure 2 we show a possible design of a MC-based acousto-optic chip. The device can be used for optical modulation and switching [5].

Because the main optical stop gap $\Delta_{\mathrm{ac}}^{\mathrm{MC}} \propto \sqrt{I_{\mathrm{SAW}}}$ follows the acoustic intensity, the dynamical response of the resonant acousto-optic Stark effect is mainly determined by a time required to build up the coherent acoustic field. The use of a SAW is favourable for the reduction of the above time, because the wave is driven through its electric component. In this case the piezoelectric coupling between excitons, the SAW, and an interdigital transducer reduces the switching on/off times of the SAWinduced Stark effect to a sub-nanosecond time scale.

\section{CONCLUSIONS}

In this paper we have developed resonant acoustooptics of MC polaritons. The following conclusions summarize our results.

(i) The giant acousto-optic nonlinearities we propose and calculate are due to virtual QW excitons which resonantly mediate the interaction of coherent (surface) acoustic phonons with MC photons.

(ii) The acoustically-induced Stark effect gives rise to the optical band gaps in the MC polariton dispersion. The gaps open up and develop with increasing intensity of the applied acoustic field and drastically change the reflection/transmission spectrum associated with MC polaritons.

(iii) The resonant acousto-optic Stark effect can be realized in standard GaAs-based microcavities parametrically driven by a SAW of the frequency $\nu_{\mathrm{ac}} \lesssim 2-3 \mathrm{GHz}$ and of the moderate intensity $I_{\mathrm{SAW}} \sim 0.1-10 \mathrm{~mW} / \mathrm{mm}$. The SAWs with the above characteristics are experimentally accessible.

(iv) The use of the acoustic pump and optical probe practically does not lead to the generation of real excitations (QW excitons) and, therefore, to the increase of the scattering rates. In contrast, the latter effect, which is proportional to the density of photo-generated real, incoherent excitons and free carriers, considerably complicates a controlled realization of the optical Stark effects within the conventional scheme "optical pump - optical probe". In the meantime the absence of real photoexcitations allows us to develop the rigorous, exactlysolvable model of the acousto-optic Stark effect in semiconductor microcavities.

(v) The resonant acousto-optic effect in SAW-pumped microcavities is very promising for device applications such as tunable optical filters, optical modulators, etc. 


\section{ACKNOWLEDGMENTS}

We appreciate valuable discussions with L V Keldysh, L E Smallwood, A V Soroko, V I Talyanskii and E A Zhukov. Support of this work by the EU RTN Project HPRN-CT-2002-00298 is gratefully acknowledged.

[1] Sapriel J 1979 Acousto-Optics (New York: Wiley \& Sons)

[2] Wilson J and Hawkes J F B 1983 Optoelectronics (London: Prentice Hall International)

[3] Korpel A 1997 Acousto-Optics (New York: M Dekker)

[4] Ivanov A L and Littlewood P B 2001 Phys. Rev. Lett. 87 136403

[5] Ivanov A L and Littlewood P B 2001 Patent GB 0121448.5

[6] Weisbuch C, Nishioka M, Ishikawa A and Arakawa Y 1992 Phys. Rev. Lett. 693314

[7] Savasta S and Girlanda R 1996 Phys. Rev. Lett. 774736

[8] Le Si Dang, Heger D, André R, Boeuf F and Romestain R 1998 Phys. Rev. Lett. 813920

[9] Savvidis P G, Baumberg J J, Stevenson R M, Skolnick M S, Whittaker D M and Roberts J S 2000 Phys. Rev. Lett. 841547

[10] Boeuf F, André R, Romestain R, Le Si Dang, Péronne F, Lampin J F, Hulin D and Alexandrou A 2000 Phys. Rev. B 62 R2279

[11] Ciuti C, Schwendimann P, Deveaud B and Quattropani A 2000 Phys. Rev. B 62 R4825

[12] Borri P, Langbein W, Woggon U, Jensen J R and Hvam J M 2000 Phys. Rev. B 63035307

[13] Baumberg J J 2002 Phys World 1537

[14] Pawlis A, Khartchenko A, Husberg O, As D J, Lischka K and Schikora D 2002 Solid State Commun. 123235

[15] Fröhlich D, Nöthe A and Reimann K 1985 Phys. Rev. Lett. 551335

[16] Von Lehmen A, Zucker J E, Heritage J P and Chemla D S 1986 Opt. Lett. 11609

[17] Mysyrowicz A, Hulin D, Antonetti A, Migus A, Masselink W T and Morkoc H 1986 Phys. Rev. Lett. 562748

[18] Combescot M and Combescot R 1988 Phys. Rev. Lett. 61117

[19] Ivanov A L and Panashchenko V V 1989 Pis'ma Zh. Eksp. Teor. Fiz. 4934 [JETP Lett. 49 39]

[20] Hulin D and Joffre M 1990 Phys. Rev. Lett. 653425

[21] Shimano R and Kuwata-Gonokami M 1994 Phys. Rev. Lett. $\mathbf{7 2} 530$

[22] Ivanov A L 1986 Zh. Eksp. Teor. Phys. 90158 [Sov. Phys. JETP 63 90]

[23] Greene B I, Mueller J F, Orenstein J, Rapkine D H, Schmitt-Rink S and Thakur M 1988 Phys. Rev. Lett. 61 325

[24] Kiseleva E S and Moskalenko S A 1999 Phys. Status Solidi (b) $\mathbf{2 1 1} 693$

[25] Hanke H, Fröhlich D, Ivanov A L, Littlewood P B and Stolz H 1999 Phys. Rev. Lett. 834365
[26] Hanke H, Fröhlich D, Ivanov A L, Littlewood P B and Stolz H 2000 Phys. Status Solidi (b) 221287

[27] Ulbrich R G and Weisbuch C 1977 Phys. Rev. Lett. 38 865

[28] Willett R L, Ruel R R, West K W and Pfeiffer L N 1993 Phys. Rev. Lett. 713846

[29] Willett R L, Ruel R R, Paalanen M A, West K W and Pfeiffer L N 1993 Phys. Rev. B 477344

[30] Shilton J M, Talyanskii V I, Pepper M, Ritchie D A, Frost J E F, Ford C J B, Smith C G and Jones G A C 1996 J. Phys: Condens. Matter 8 L531

[31] Cunningham J, Talyanskii V I, Shilton J M, Pepper M, Kristensen A and Lindelof P E 2000 Phys. Rev. B 62 1564

[32] Rocke C, Zimmermann S, Wixforth A and Kotthaus J P 1997 Phys. Rev. Lett. 784099

[33] Rocke C, Govorov A, Wixforth A, Böhm G and Weimann G 1998 Phys. Rev. B 57 R6850

[34] Govorov A O, Kalameitsev A V, Rotter M, Wixforth A, Kotthaus J P, Hoffmann K-H and Botkin N 2000, Phys. Rev. B 622659

[35] Yu P Y and Cardona M 1996 Fundamentals of Semiconductors (Berlin: Springer) ch 3

[36] Zeldovich Ya B 1966 Zh. Eksp. Teor. Phys. 511492 [1967 Sov. Phys. JETP 24 1006]

[37] Ivanov A L, Littlewood P B and Haug H 1999 Phys. Rev. $B \mathbf{5 9} 5032$

[38] Nägerl J S, Stabenau B, Böhne G, Dreher S, Ulbrich R G, Manzke G and Henneberger K 2001 Phys. Rev. B 63 235202

[39] The one dimensional geometry of nonresonant acoustooptic interaction is proposed for high-quality (GaAs) superlattices driven by a bulk acoustic wave of frequency $\nu_{\text {ac }} \lesssim 1 \mathrm{GHz}$ (He J and Sapriel J 1989 Appl. Phys. Lett. 55 2292)

[40] Campbell C 1989 Surface Acoustic Wave Devices and their Signal Processing Applications (New York: Academic)

[41] Östling D and Engan H E 1995 Opt. Lett. 201247

[42] Farnell G W 1978 Acoustic Surface Waves ed A A Oliner Topics in Applied Physics Vol 24 (Berlin: Springer) ch 2

[43] Hess P 2002 Physics Today 5542

[44] Malpuech G, Di Carlo A, Kavokin A, Baumberg J J, Zamfirescu M and Lugli P 2002 Appl. Phys. Lett. 81412 

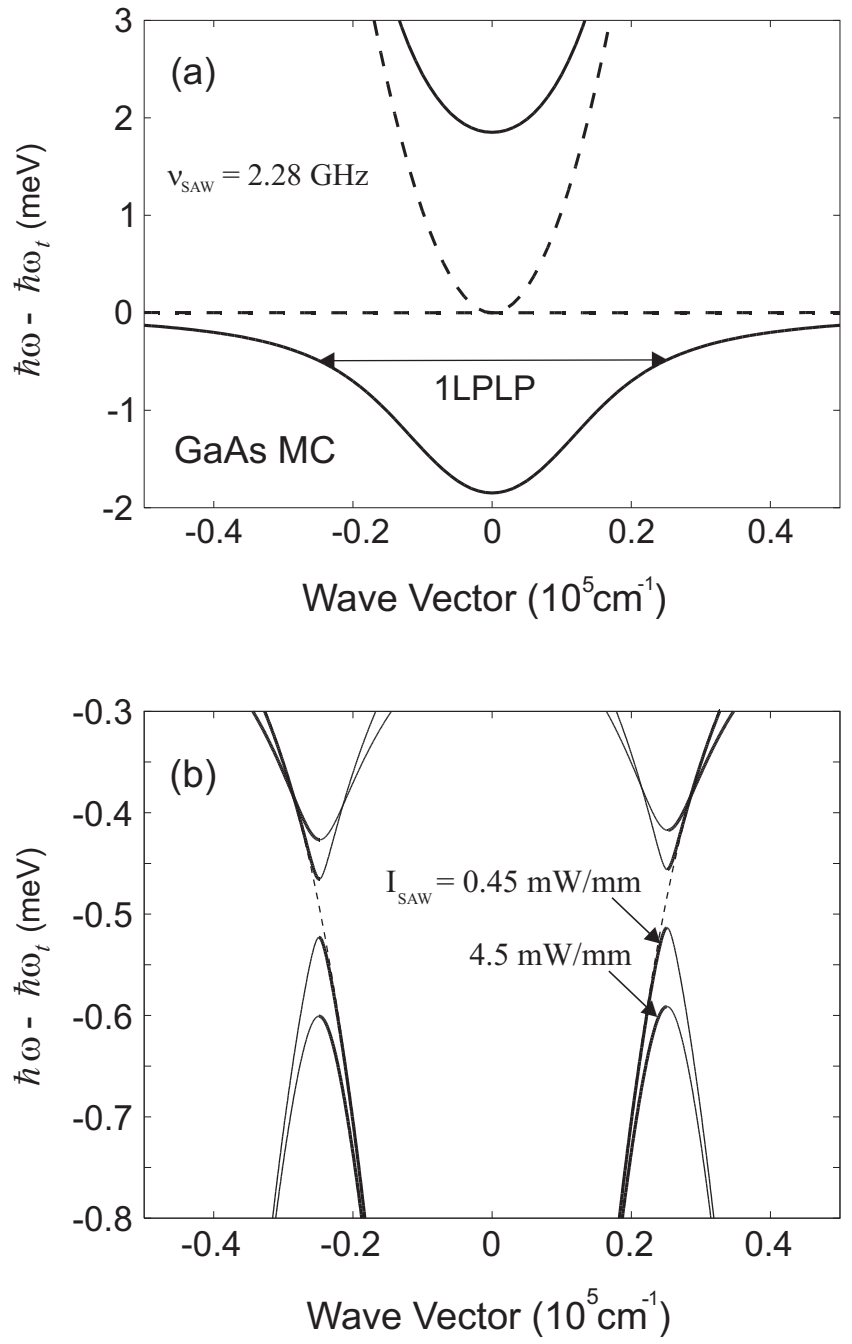

FIG. 1. An acoustically-pumped GaAs-based microcavity with the Rabi splitting energy of $\mathrm{MC}$ polaritons $\hbar \Omega_{\mathrm{X}}^{\mathrm{MC}}=3.7 \mathrm{meV}$ and zero detuning, i.e. $\omega_{0}=\omega_{t}$. The (surface) acoustic wave is characterized by in-plane $|\mathbf{k}|=5 \times 10^{4} \mathrm{~cm}^{-1}$ and $\nu_{\mathrm{ac}}=\nu_{\mathrm{SAW}}=2.28 \mathrm{GHz}$. (a) The in-plane QW exciton and MC photon dispersions (dashed lines), and the $\mathrm{MC}$ polariton dispersion branches (solid lines) calculated by equation (6). The arrow 1LPLP indicates one-phonon resonant transition between two counter-propagating lower-branch MC polaritons with in-plane wavevectors $\mathbf{p}_{\|}= \pm \mathbf{k} / 2$. (b) The acoustically-induced quasi-energy spectrum calculated by equation (4) for a spectral vicinity of the first optical band gap. The acoustic intensity is given by $I_{\mathrm{SAW}}=0.45$ and $4.5 \mathrm{~mW} / \mathrm{mm}$. The thick solid lines visualize the acoustically-induced gap in MC polariton spectrum.

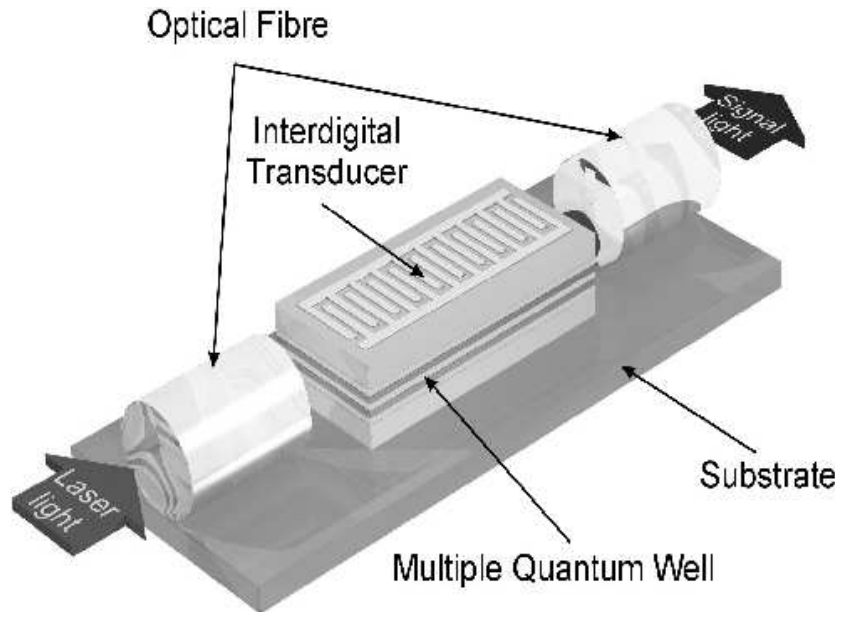

FIG. 2. Schematic of a SAW-driven semiconductor microcavity for optical modulation and switching. The surface acoustic wave with in-plane wavevector $\mathbf{k}$ resonantly couples two counter-propagating MC polariton states, $\left\{\mathbf{p}_{\|}=\mathbf{k} / 2, \omega^{\mathrm{MC}}(k / 2)\right\}$ and $\left\{\mathbf{p}_{\|}=-\mathbf{k} / 2, \omega^{\mathrm{MC}}(k / 2)\right\}$. The probe light field has frequency $\omega \simeq \omega^{\mathrm{MC}}(k / 2)$. 